\title{
Proceso constructivo del cimorro de la Catedral de Ávila (siglos XII-XIV). Hipótesis verificadas a partir del análisis de estabilidad estructural
}

\author{
The constructive process in the Cimorro zone in the the Cathedral of Avila, \\ (century XII-XIV). Hypothesis and its verification through the analysis \\ of Structural Stability Smart and Sustainable Offices (SSO)
}

\author{
$\underline{\mathrm{M}}^{\mathrm{a}} \mathrm{A}$. Benito Pradillo ${ }^{(*)}$
}

\section{RESUMEN}

En este artículo se presenta una hipótesis del proceso constructivo seguido en el Cimorro de la Catedral de Ávila, durante las tres etapas de su construcción, correspondientes a los siglos XII, XIII y XIV, respectivamente. Las hipótesis propuestas se han verificado mediante análisis de estabilidad, en concreto, del Análisis Límite para Estructuras de Fábrica. Después de comprobar la estabilidad estructural para cada una de las etapas constructivas se realizan las siguientes afirmaciones: La bóveda central se construyó y cerró en el siglo XII, careciendo durante un breve periodo de tiempo de sistema de contrarresto alguno, hasta la colocación de la tribuna. En el siglo XIII se aumentó la fortificación mediante la barbacana y dos adarves. En la centuria siguiente, siglo XIV, se desmontó la tribuna para dotar al Cimorro del sistema de contrarresto con estribo y dos arbotantes, que podemos ver actualmente.

Palabras clave: Mecánica de fábricas, análisis límite, estructura gótica, sistemas de contrarresto, catedral de Ávila, arquitectura gótica.

\section{ABSTRACT}

This paper presents a hypothesis about the order in the constructive process for the head zone in the Cathedral of Ávila, called Cimorro. The hypothesis proposed in this area considers for its construction three different stages: XII Century, XIII Century and XIV Century. The proposed hypotesis have been verified with the Analysis of Stability realised. The theoretical Framework used is the Limit Analysis to Masonry Structures. After prooving the Structural Stability for each of the building stages, the following conclusions are made: Through the XII Century, the possibility of closing the central vault without Buttress System for a short period of time and the subsequent placement of a tribune is stated. A fortification formed by the barbican and the «adarves» were built during the XIII Century. In the XIV Century, the tribune is removed and replaced with the Buttress System with buttress and double flying buttress, which we can see nowadays.

Keywords: Mansonry structure, analysis of Structural Stability, Gothic architecture, Avila Cathedral, system battlement.

(*) Departamento de Mecánica de Medios Continuos y Teoría de Estructuras. Universidad Politécnica de Valencia (España). Persona de contacto/Corresponding author: mabenitopradillo@gmail.com ( $\mathrm{M}^{\mathrm{a}} \mathrm{A}$. Benito Pradillo)

ORCID: http://orcid.org/oooo-0001-6704-6394 (Ma A. Benito Pradillo)

Cómo citar este artículo/Citation: Benito Pradillo, Ma A. (2017). Proceso constructivo del cimorro de la Catedral de Ávila (siglos XII-XIV). Hipótesis verificadas a partir del análisis de estabilidad estructural. Informes de la Construcción, 69(548): e227, doi: http://dx.doi.org/10.3989/ic.16.047.

Copyright: (C) 2017 CSIC. Licencia / License: Salvo indicación contraria, todos los contenidos de la edición electrónica de Informes de la Construcción se distribuyen bajo una licencia de uso y distribución Creative Commons Attribution License (CC BY) Spain 3.o. 


\section{OBJETO DE ESTUDIO Y MARCO DE LA INVESTIGACIÓN}

La Catedral de Ávila se inició en el siglo XII a partir de su cabecera, sobre cuya planta románica se reajustó una estructura gótica, y a la que en el siglo XIII se añadió el cuerpo de naves, ya en estilo plenamente gótico, dando lugar a una de las primeras catedrales góticas españolas.

Se trata de una catedral-fortaleza que, condicionada por la orografía de la ciudad y su amurallamiento, se concibió como bastión militar; este hecho queda especialmente patente el Cimorro, parte exterior de la girola que, embutida en la muralla, constituye el cubo mayor del recinto amurallado (1) (fig. 1).

Debido a la falta de fuentes documentales, la datación de la cabecera y su evolución constructiva entre los siglos XII y XIV han sido objeto de discrepancias por parte de la historiografía previa, en concreto, recientemente, por Gutiérrez (2000) (2), Navascués (2004) (3) y Martín (2006)(4): Martín 2006 (2), Navascués 2004 (3) y Gutiérrez 2000 (4). El análisis del edificio y la muralla puede contribuir a resolver las lagunas al respecto; este artículo pretende clarificar el proceso constructivo a partir del análisis estructural del Cimorro. El trabajo, abordado por la autora de este artículo en su Tesis Doctoral sobre la evolución constructiva y el análisis estructural de la
Catedral de Ávila, UPM 2011 (11),se desarrolló en el marco del proyecto de investigación financiado por la Universidad Católica de Ávila (2009-2011) con el título Análisis de técnicas métricas de modelado $3 \mathrm{~d}$ : aplicación a la reconstrucción virtual del Cimorro de la Catedral de Ávila, liderado por la Dra. Madrid de la Fuente.

\section{METODOLOGÍA DE ANÁLISIS}

Este estudio se ha realizado a partir de una exhaustiva y rigurosa toma de datos, como fundamento de la restitución geométrica del conjunto y de su análisis estructural (5), en el marco de la tecnología constructiva de cada época (6).

La toma de datos se ha desarrollado a partir de fuentes bibliográficas, trabajo de archivo y un intenso trabajo de campo. La labor de archivo se ha centrado en las referencias concernientes a las obras acometidas en la cabecera entre los siglos XII y XIV, recogidas (7) en las Actas Capitulares, los Libros de Cuentas, los Libros de Consistorio, protocolos, informes y contratos de obras, pergaminos y códices. El trabajo de campo ha consistido en la lectura de las patologías (deformaciones y grietas) de las fábricas y las bóvedas y la medición de los desplomes.

A partir de estos datos y de la planimetría recopilada, las representaciones iconográficas de la catedral y sus fotografías

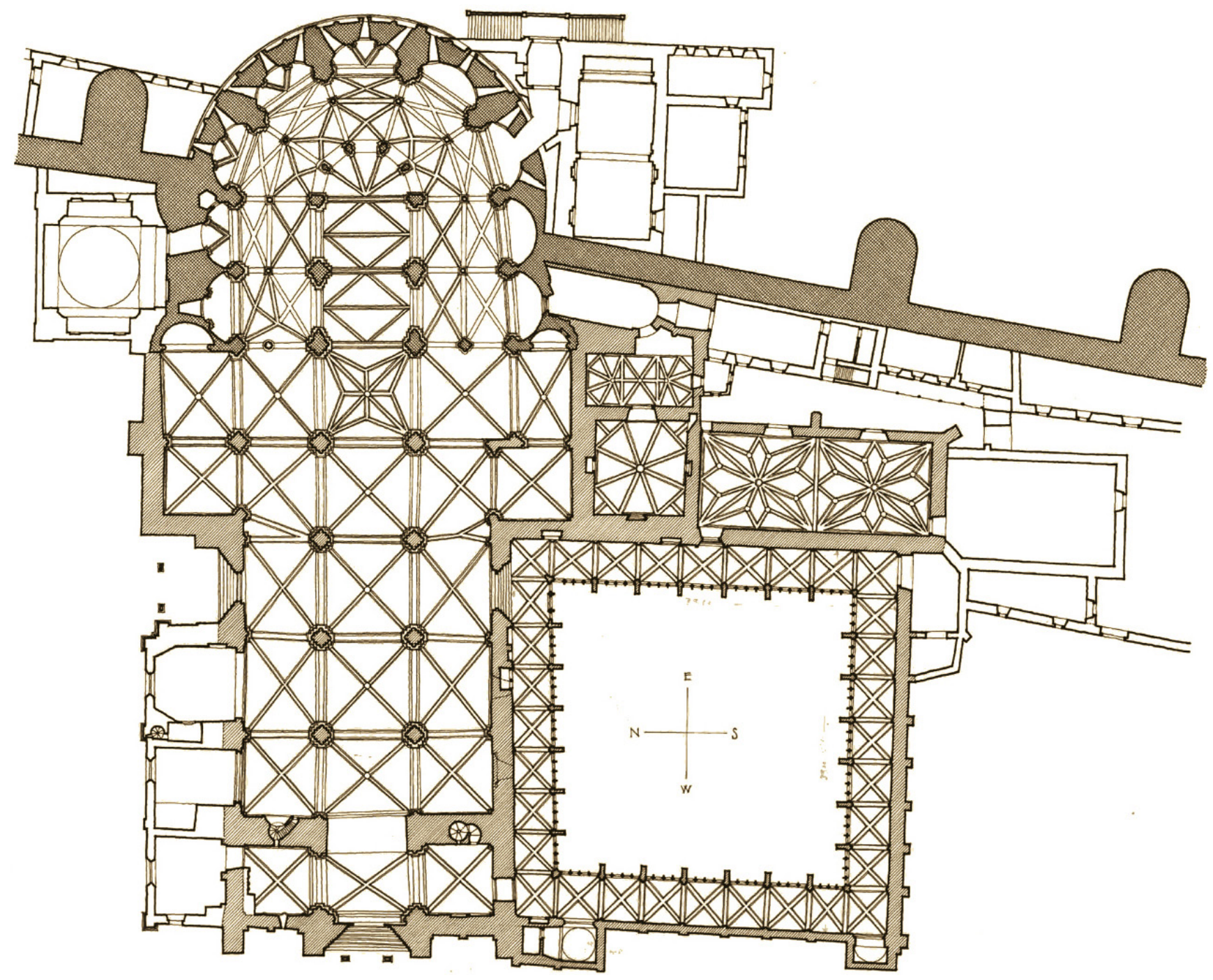

Figura 1. Planta de la Catedral de Ávila (Merino, 1981). 

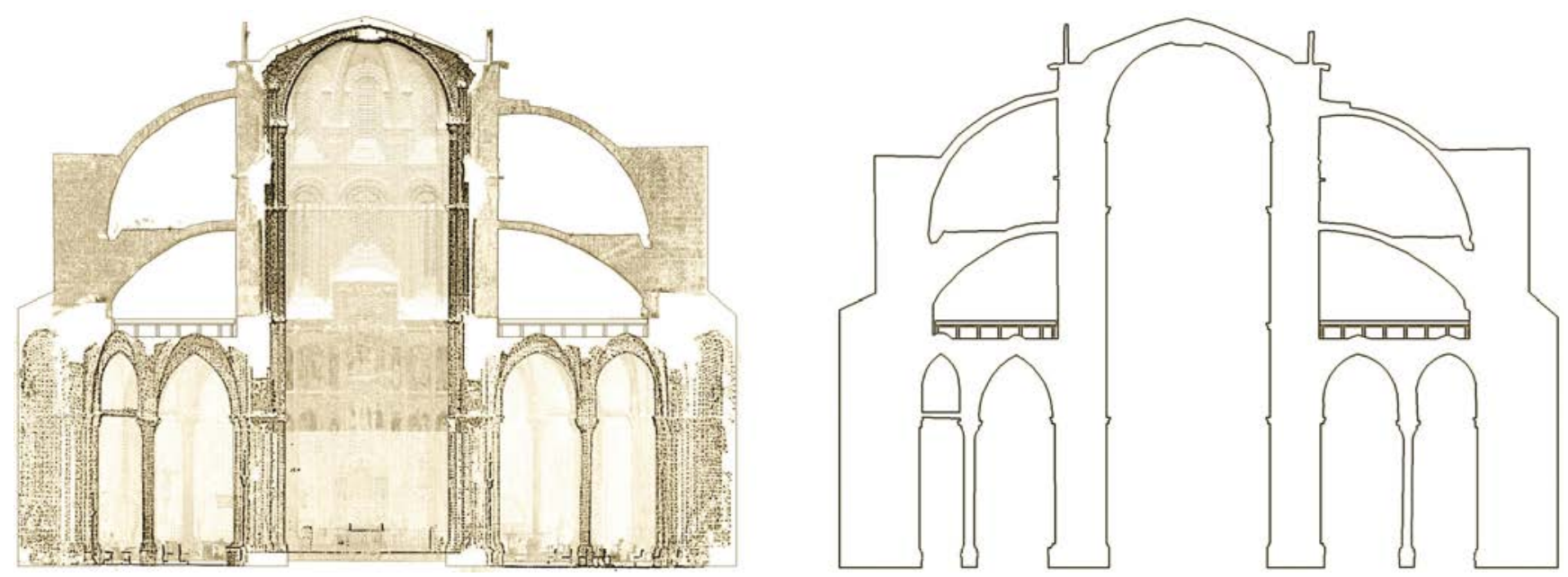

Figura 2. Datos procedentes de las nubes de puntos y restitución gráfica de la sección real en línea (Benito, 2009).

históricas, se ha realizado un levantamiento (8) mediante un escaneado laser $3 \mathrm{~d}$ con posicionamientos tanto exteriores en el Cimorro (incluyendo los arbotantes y la barbacana) como interiores (en la girola y el presbiterio), a fin de combinar las diferentes posiciones para conseguir una visión completa de las nubes de puntos empleada en la restitución gráfica de la planimetría en CAD. Sobre las nubes de puntos se ha dibujado la sección obteniendo una restitución de la geometría real de los elementos (fig. 2), que se ha utilizado para realizar el análisis estructural de equilibrio de la sección. El análisis de la sección de cada etapa constructiva ha permitido verificar la validez de las hipótesis propuestas. El marco teórico del método utilizado para el análisis de la estabilidad estructural es el Análisis Límite de Estructuras de Fábrica, desarrollado por Heyman (9).

\section{PROCESO CONSTRUCTIVO}

Se parte de la hipótesis de que la bóveda del presbiterio se construyó en el siglo XII, quedando cerrada durante un breve periodo de tiempo sin ningún sistema de contrarresto (fig. 3.a), y que la colocación de la tribuna fue posterior (fig. 3.b). Una vez construida la fortificación (la barbacana y los adarves) en la centuria posterior (fig. 3.c), se mantuvo la tribuna. Por último, en el siglo XIV se desmontó la tribuna, para dotar al Cimorro del sistema de contrarresto definitivo, con estribo y dos arbotantes (fig. 3.d), que hoy se mantiene.

\subsection{Fase 1. Siglo XII}

Durante la primera campaña constructiva del primer proyecto cisterciense de la Catedral, correspondiente al siglo XII, se realizó la cabecera con sus capillas absidiales y el basamento de granito que las unía.

Según Rodríguez Almeida (10), las capillas absidiales estarían vistas al exterior, siendo posterior el muro que las abraza; el autor, indica también la existencia de restos de este primer basamento, claramente diferenciados respecto al resto de la fábrica (fig. 4).

Una de las hipótesis de partida es que en el siglo XII en un breve espacio de tiempo, pudieron darse dos situaciones, en función de que la tribuna superior estuviera o no cubierta.

Es por ello que, en primer lugar (opción a), se analiza la estabilidad de la sección con un basamento inferior, desprovista de la fortificación actual, y considerando que la tribuna superior no estuviera cubierta; se estudia el equilibrio de la estructura con la bóveda del presbiterio y las bóvedas de la girola pero sin el contrarresto de la tribuna (fig. 3, izquierda).

En segundo lugar (opción b), se analiza la misma sección considerando como contrarresto el cierre de la tribuna, construida con nervios y plementería (fig. 5).

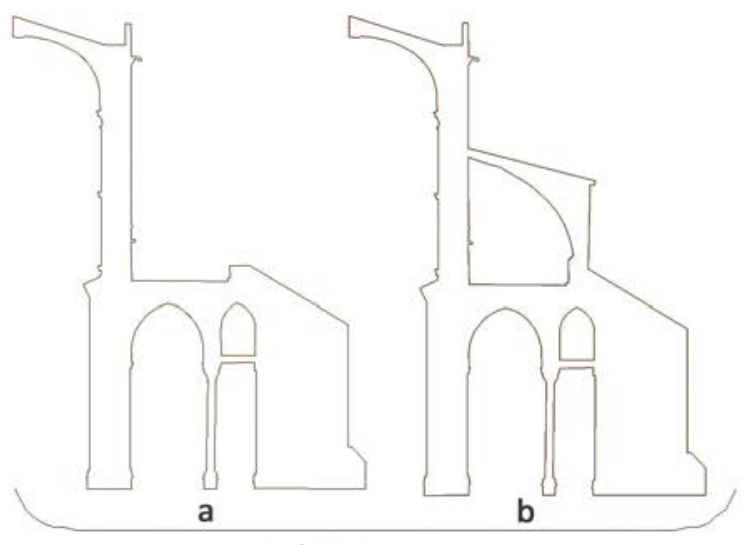

siglo XII

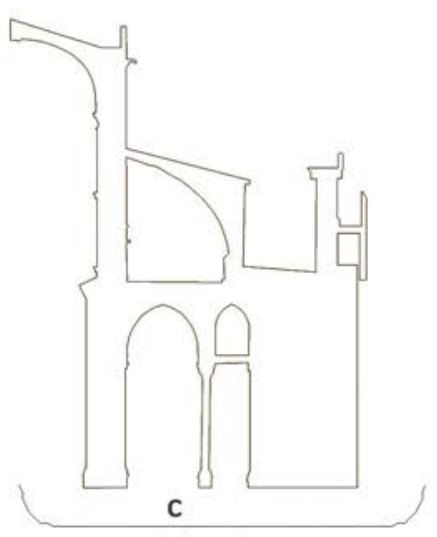

siglo XIII

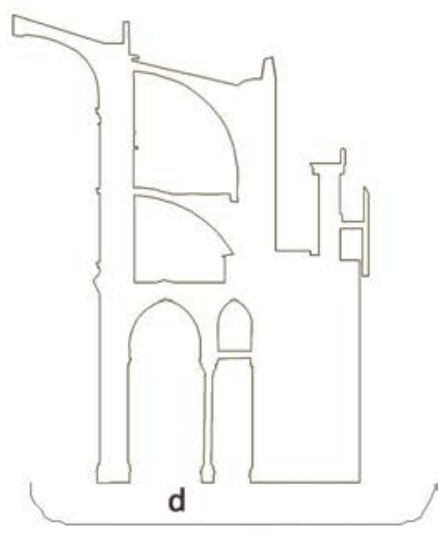

siglo XIV

Figura 3. Evolución constructiva del Cimorro, a partir de su sección radial (Benito, 2011). 


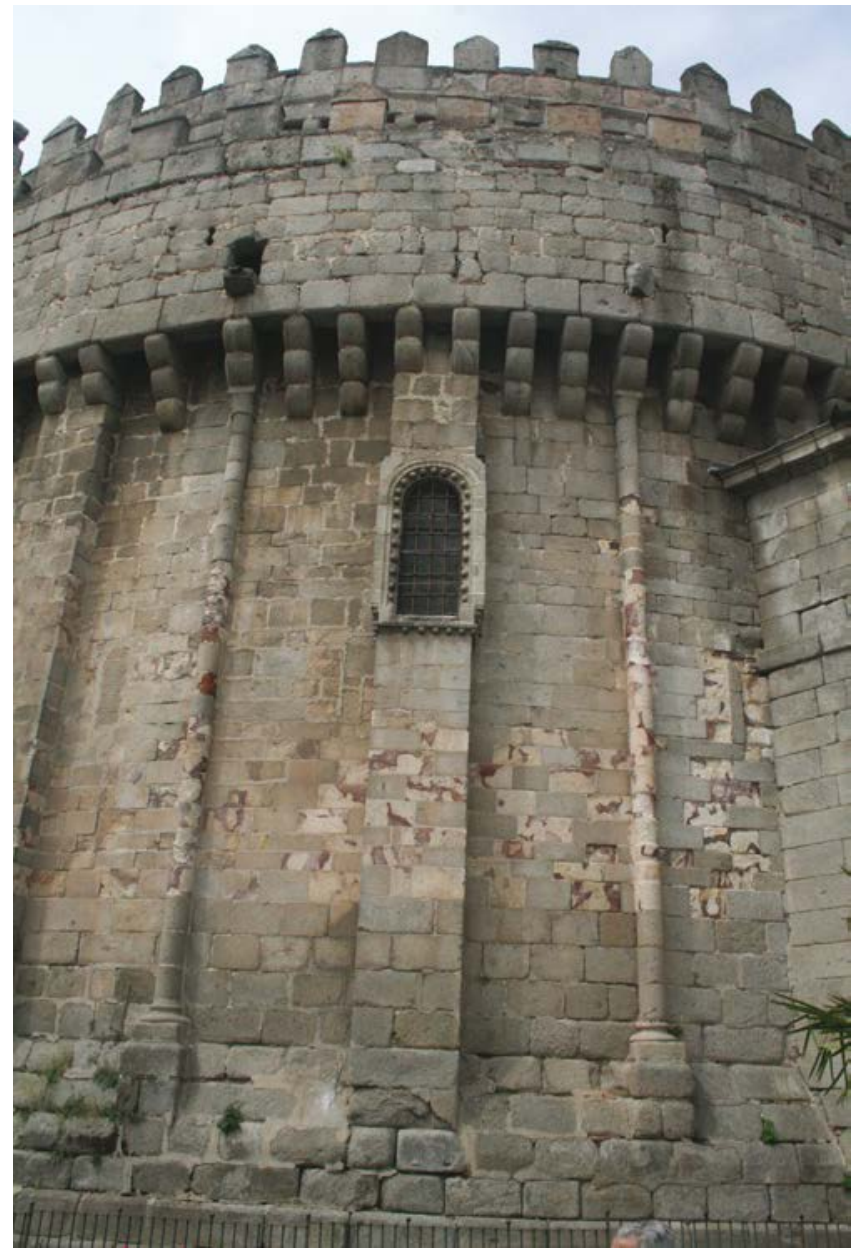

Figura 4. Exterior del Cimorro de la Catedral de Ávila.

\subsection{Fase 2. Siglo XIII}

La fortificación de la cabecera se ejecutó en dos etapas diferentes. La primera, finales del siglo XII, cuando el maestro Don Varón fortificó los absidiolos incorporándole un forro exterior cilíndrico.

La segunda, a mediados del siglo XIII, cuando se añadió un forro de sillería hasta la altura del adarve de la muralla y se construyó la barbacana (fig. 6).

\subsection{Fase 3. Siglo XIV}

En la primera mitad del siglo XIX, se retiraron los nervios de la tribuna y se colocaron los arbotantes (fig. 7). Esta intervención se realizó bajo el mecenazgo del obispo Sancho Blázquez Dávila (1312-1356), sin duda a consecuencia de los graves problemas estructurales que, según refleja la documentación, la catedral presentaba en 1319: "La catedral de Sant Salvador de Ávila, nuestra madre, estaba en gran peligro assi que si no fuesse acorrida mucho ayna estava en tiempo de se perecer»

Otra actuación del momento, fue la modificación de la cubierta, con pendiente a un agua, y la sustitución de la techumbre pétrea por una de madera y teja.

Mientras el sistema de cubierta fue cambiado posteriormente, ya que en el siglo XVI se realizó una cubierta a dos aguas para abrir los ventanales de la zona inferior del presbiterio, el sistema estructural de contrarresto no varió; de hecho, éste se conservó hasta que en2001 se realizó una cubierta plana y se volvió a utilizar la tribuna como galería exterior.
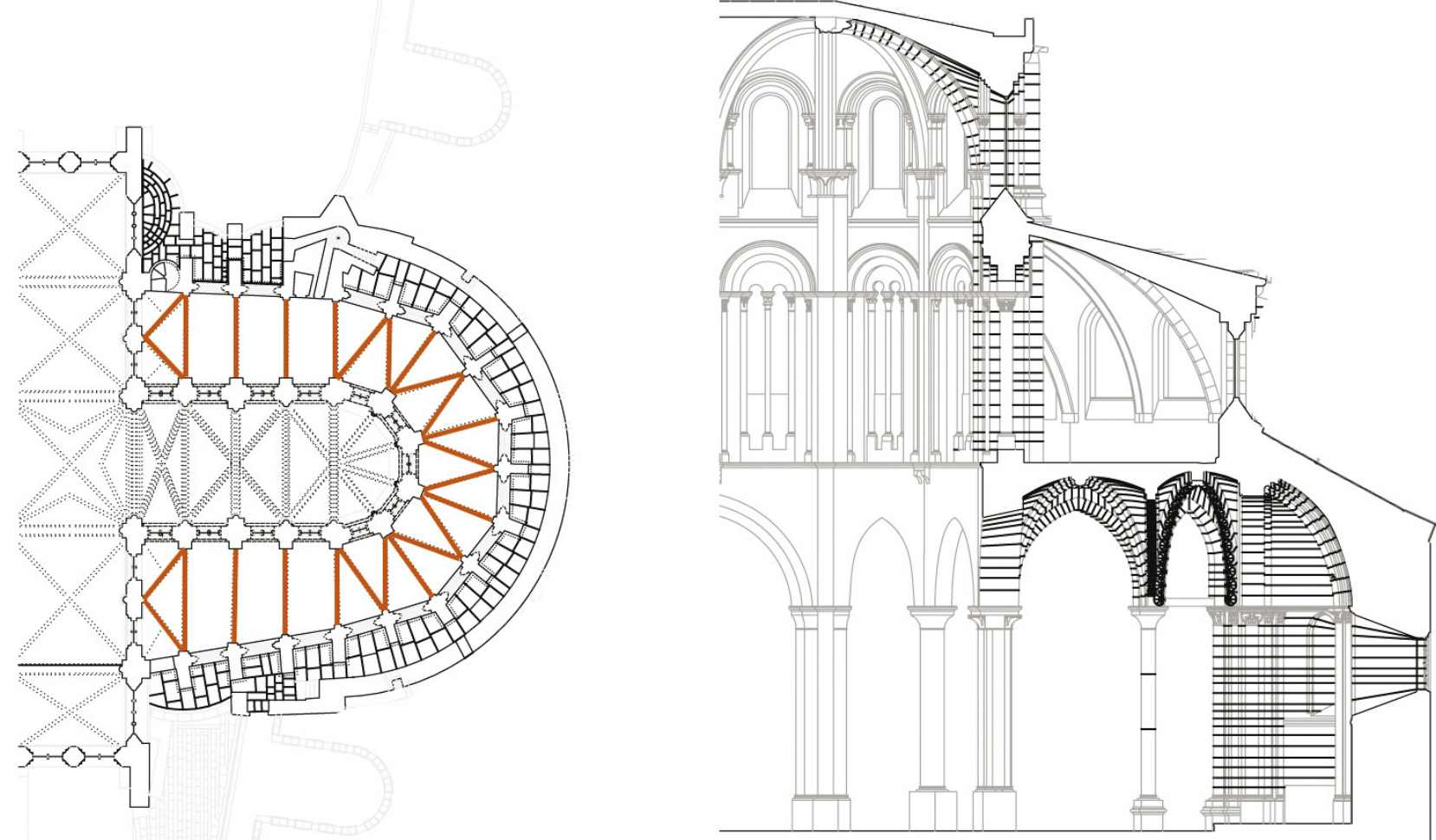

Figura 5. Campaña constructiva del siglo XII (planta y sección) (Benito, 2016). 

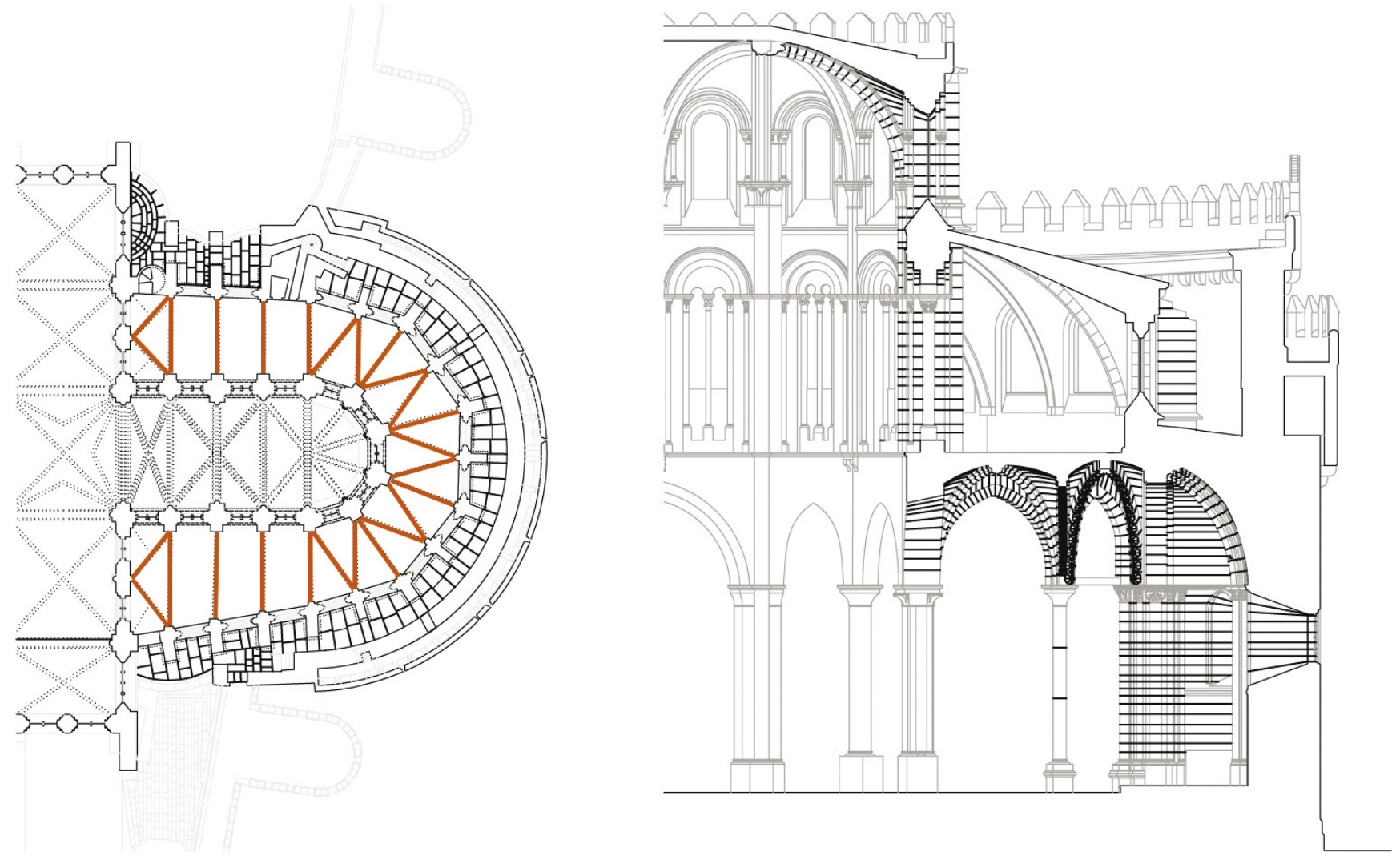

Figura 6. Campaña constructiva del siglo XIII (planta y sección) (P. Feduchi, 1998).
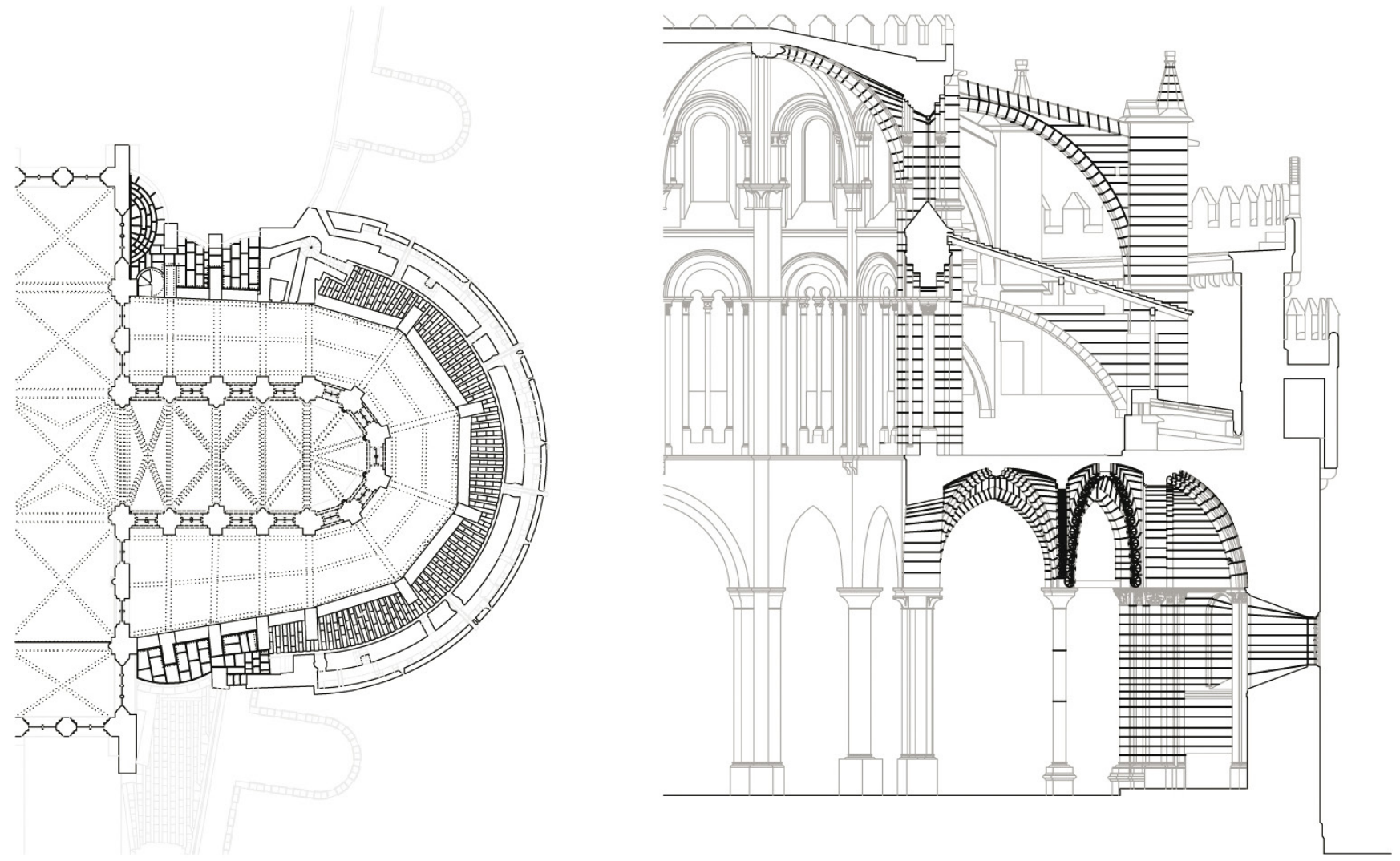

Figura 7. Campaña constructiva del siglo XIV (planta y sección) (P. Feduchi, 1998). 


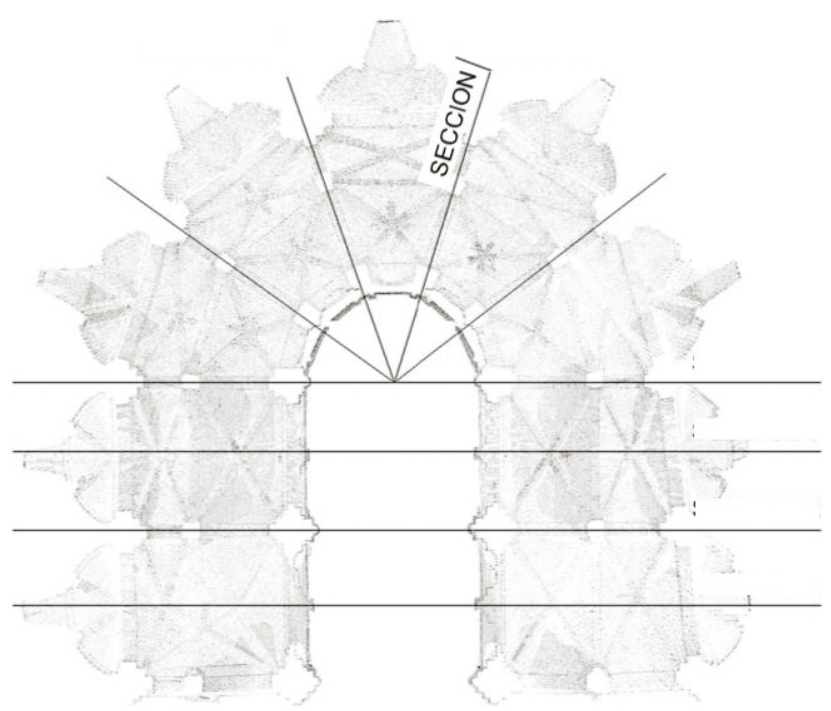

Figura 8. Planta de la cabecera de la Catedral de Ávila (Benito, 2009).

El análisis realizado se limita al siglo XIV, dado que entonces se colocaron los arbotantes, se realizaron modificaciones estructurales sustanciales que afectaran al equilibro estructural del edificio, ya que las intervenciones posteriores solo modificaron la geometría de su cubierta.

\section{VERIFICACIÓN DE LAS HIPÓTESIS}

El equilibrio de la sección gótica es interesante, no sólo en el estado final sino también durante el proceso de construcción. Por ello el estudio del análisis de estabilidad estructural se considera criterio para la verificación de la hipótesis planteada para el proceso constructivo.

A fin de realizar este análisis, se ha elegido la sección radial por la cabecera (fig. 8); los valores de las fuerzas utilizados se encuentran explicitados con detalle en la tesis doctoral de la autora (12).
En los cuatro casos, el análisis ha consistido en enumerar las cargas que afectan a la sección, calcular la intersección de la LDE con unos planos de corte previamente elegidos, dibujar una envolvente de las líneas de empujes, e interpretar y valorar los resultados, indicando el coeficiente de seguridad geométrico en el pilar y en el estribo.

\subsection{Análisis de la fase 1 (siglo XII, opción A)}

La sección tiene un basamento perimetral uniendo las capillas absidiales y no existe la tribuna. Para las fuerzas, se han utilizado los valores recogidos en la tabla 1.

La sección se divide en bloques y se suman las fuerzas en cada bloque para hallar la intersección de la resultante con el plano de corte. Por esos puntos pasa la línea de empujes (fig. 9).

Tabla 1. Valores de cálculo para fase 1 (siglo XII, opción a) (Benito, 2011).

\begin{tabular}{|c|c|c|c|}
\hline & Elemento & $\begin{array}{c}\text { Componente } \\
\text { vertical KN }\end{array}$ & $\begin{array}{l}\text { Componente } \\
\text { horizontal KN }\end{array}$ \\
\hline $\mathrm{F} 1$ & Pilar $(\mathrm{h}=2.42)$ & 151.44 & o \\
\hline F2 & Bóveda presbiterio & 286.48 & 63.69 \\
\hline F3 & Pilar (h=10.74) & 672.1 & O \\
\hline $\mathrm{F}_{4}$ & $\begin{array}{l}\text { Bóveda girola } \\
\text { interior }\end{array}$ & 229.91 & 33.45 \\
\hline $\mathrm{F}_{5}$ & Pilar $(\mathrm{h}=11.53)$ & 721.54 & $\mathrm{O}$ \\
\hline F6 & $\begin{array}{l}\text { Bóveda girola } \\
\text { interior }\end{array}$ & 229.91 & $33 \cdot 45$ \\
\hline F7 & $\begin{array}{c}\text { Bóveda girola } \\
\text { exterior }\end{array}$ & 320.15 & 58.64 \\
\hline F8 & Pilar interior girola & 28.99 & $\mathrm{O}$ \\
\hline F9 & Codal de piedra & o & 25.19 \\
\hline F10 & $\begin{array}{c}\text { Bóveda girola } \\
\text { exterior }\end{array}$ & 320.15 & 58.64 \\
\hline F11 & $\begin{array}{l}\text { Muro exterior } \\
(\mathrm{h}=3.75)\end{array}$ & 1190.7 & $\mathrm{O}$ \\
\hline F12 & $\begin{array}{l}\text { Muro exterior } \\
(\mathrm{h}=7.0)\end{array}$ & 2226.4 & $\mathrm{o}$ \\
\hline
\end{tabular}

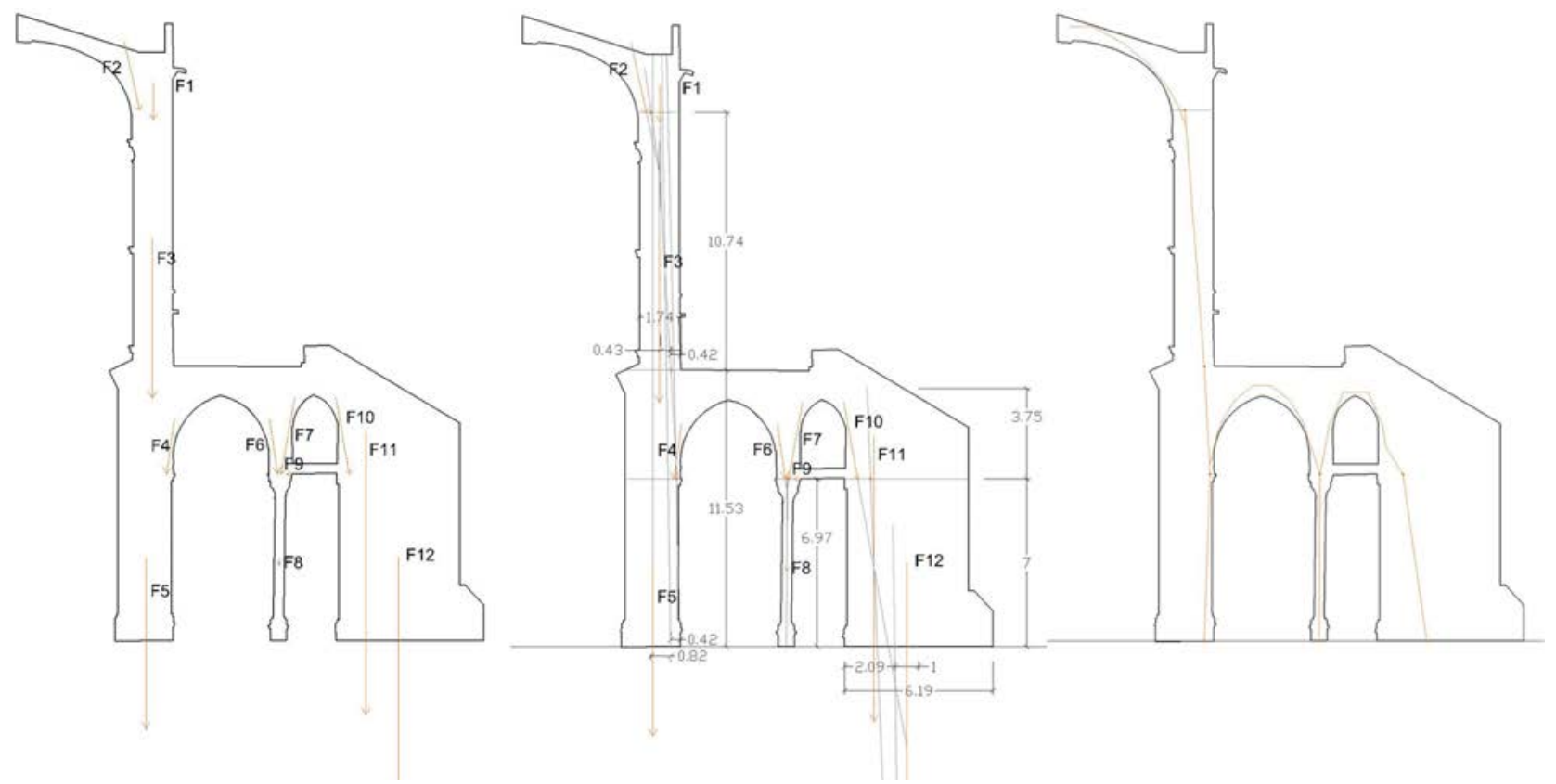

Figura 9. Distribución de fuerzas y LDE en la sección radial por el Cimorro en la opción a del siglo XII (Benito, 2011). 
Análisis en la zona del pilar

En la zona superior del pilar, el empuje de la bóveda del presbiterio no está contrarrestado, por lo que la LDE queda descentrada. El punto de intersección de la LDE en esta sección, se sitúa a $42 \mathrm{~cm}$ del borde exterior derecho y a $43 \mathrm{~cm}$ del centro, resultando fuera del tercio central.

En la zona inferior, la distancia de la intersección LDE con la base del pilar al borde exterior derecho es de $42 \mathrm{~cm}$, quedando a $82 \mathrm{~cm}$ del centro, se sitúa también fuera del tercio central.

Dado que la LDE está incluida dentro de la sección, la situación es estable; sin embargo, al no pasar por el tercio central del pilar interior, el coeficiente de seguridad geométrica queda reducido.

\section{Análisis en la zona del estribo}

La intersección de la LDE con la base del estribo está incluida en tercio central de la sección. El punto de paso de la LDE se sitúa hacia el interior de la sección y separado $209 \mathrm{~cm}$ del extremo izquierdo del estribo (fig. 9).

\subsection{Análisis de fase 1 (siglo XII, opción B)}

La sección tiene un basamento perimetral uniendo las capillas absidiales y tribuna sobre la girola. Para las fuerzas, se han utilizado los valores recogidos en la tabla 2.

La opción b que se plantea para esta campaña es la construcción de una tribuna cubierta con una bóveda apoyada en unos nervios (fig. 6). Para contrarrestar el empuje de la bóveda del presbiterio en la zona curva tenemos tres nervios. Para el cálculo sumamos las componentes verticales de los tres nervios, y las componentes horizontales las proyectamos en la dirección de la sección.

La sección se divide en bloques y se suman las fuerzas en cada bloque para hallar la intersección de la resultante con
Tabla 2. Valores de cálculo para fase 1 (siglo XII, opción b) (Benito, 2011).

\begin{tabular}{|c|c|c|c|}
\hline & Elemento & $\begin{array}{c}\text { Componente } \\
\text { vertical KN }\end{array}$ & $\begin{array}{c}\text { Componente } \\
\text { horizontal KN }\end{array}$ \\
\hline $\mathrm{F} 1$ & Pilar $(\mathrm{h}=2.42)$ & 151.44 & $\mathrm{O}$ \\
\hline $\mathrm{F} 2$ & Bóveda presbiterio & 286.48 & 63.69 \\
\hline F3 & $\begin{array}{c}\text { Tribuna nervio } \\
\text { superior }\end{array}$ & 7.62 & 80.81 \\
\hline $\mathrm{F} 4$ & Pilar $(h=10.74)$ & 672.1 & $\mathrm{O}$ \\
\hline $\mathrm{F}_{5}$ & $\begin{array}{c}\text { Bóveda girola } \\
\text { interior }\end{array}$ & 229.91 & $33 \cdot 45$ \\
\hline F6 & Pilar $(h=11.53)$ & 721.54 & O \\
\hline F7 & $\begin{array}{c}\text { Bóveda girola } \\
\text { interior }\end{array}$ & 229.91 & $33 \cdot 45$ \\
\hline F8 & $\begin{array}{c}\text { Bóveda girola } \\
\text { exterior }\end{array}$ & 320.15 & 58.64 \\
\hline F9 & Codal de piedra & $\mathrm{O}$ & 25.19 \\
\hline F10 & Pilar interior girola & 28.99 & O \\
\hline $\mathrm{F} 11$ & $\begin{array}{c}\text { Tribuna nervio } \\
\text { inferior }\end{array}$ & 302.1 & 30.87 \\
\hline F12 & $\begin{array}{c}\text { Bóveda girola } \\
\text { exterior }\end{array}$ & 320.15 & 58.64 \\
\hline $\mathrm{F} 13$ & $\begin{array}{l}\text { Muro exterior } \\
(\mathrm{h}=3.75)\end{array}$ & 1190.7 & o \\
\hline F14 & $\begin{array}{l}\text { Muro exterior } \\
(\mathrm{h}=7.0)\end{array}$ & 2226.4 & o \\
\hline
\end{tabular}

el plano de corte. Por esos puntos pasa la línea de empujes (fig. 10).

\section{Análisis en la zona del pilar}

El empuje de los nervios de la tribuna tiene su punto de aplicación en el punto medio del pilar, lo que sitúa la LDE centrada respecto al pilar en la zona superior (fig. 10). Para contrarrestar de forma más eficaz el empuje de las bóvedas, se considera conveniente situar el empuje de la tribuna en un punto más alto del pilar, donde en el siglo XIV se colocará el arbotante superior.
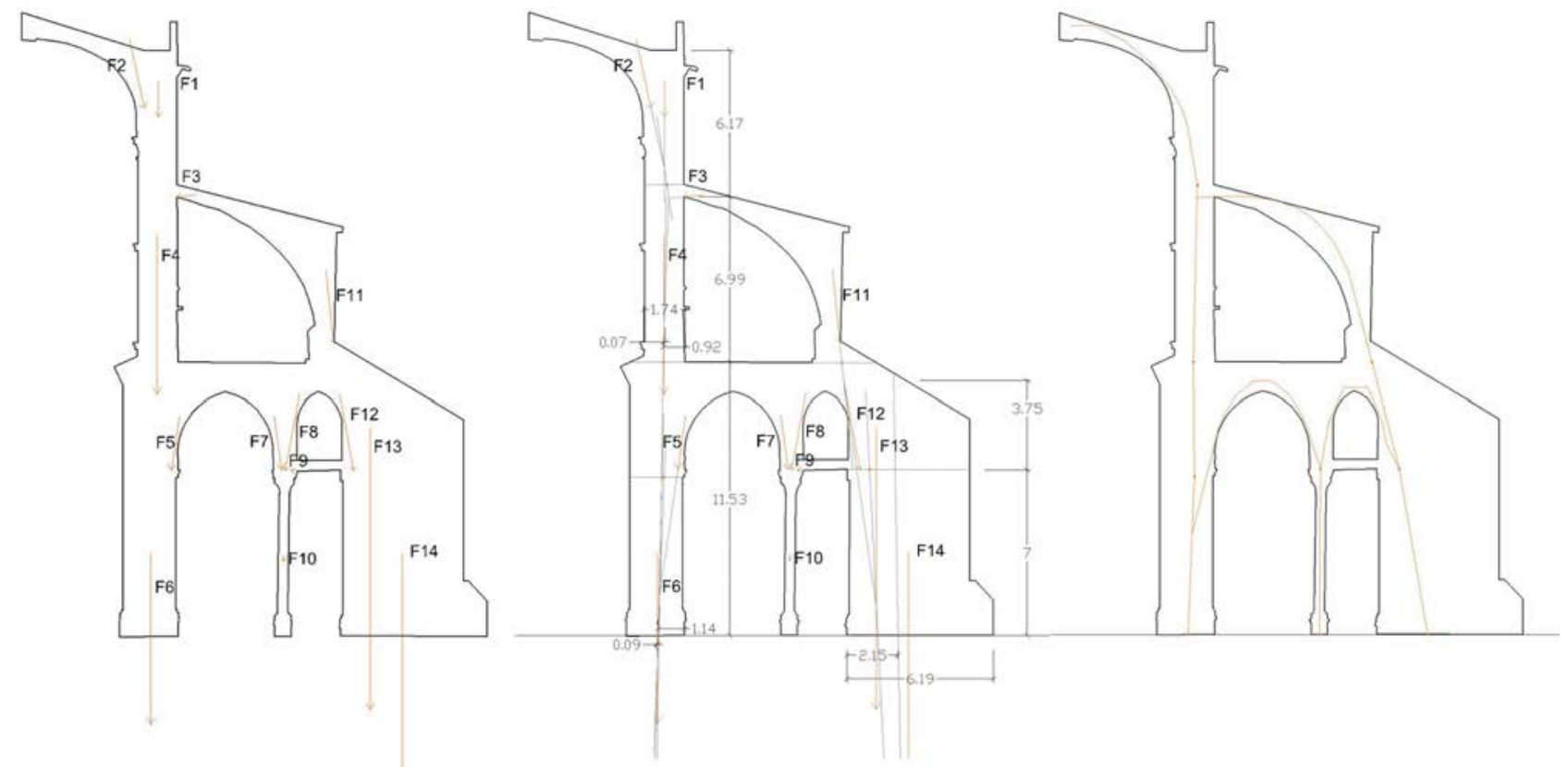

Figura 10. Distribución de fuerzas y LDE en la sección radial por el Cimorro en la opción b del siglo XII (Benito, 2011). 
En la parte superior del pilar, la LDE pasa a $7 \mathrm{~cm}$ del punto medio del pilar, y en la zona de la base del pilar a $9 \mathrm{~cm}$; en ambos casos, la resultante se incluye en el tercio central.

Análisis en la zona del estribo

En cuanto al estribo, la diferencia respecto a la opción anterior no es significativa, aunque también en este caso la LDE pasa más centrada en la base del estribo.

\subsection{Análisis de la fase 2 (siglo XIII)}

En la fase 2, correspondiente al siglo XII, la tribuna y la fortificación del doble almenado y la barbacana están construidas, presentando el estado en que actualmente se encuentran.. Para las fuerzas, se han utilizado los valores recogidos en la tabla 3.

Se divide la sección en bloques y se halla el punto de intersección de la LDE en cada sección (fig. 11).

Análisis en la zona del pilar

La intersección de la LDE con la base del pilar está separado $27 \mathrm{~cm}$ del punto medio. Siendo el ancho del pilar de 2,46 cm el coeficiente de seguridad geométrico en el pilar será de 4,5.

Análisis en la zona del estribo

En el estribo, la carga procedente de la fortificación centra la LDE en la base. El estribo tiene un espesor de 6,19 $\mathrm{m}$ y el punto de intersección de la LDE está separado del punto medio $27 \mathrm{~cm}$. El coeficiente de seguridad geométrico para el estribo es de 11,4.

\subsection{Análisis de la fase 3 (siglo XIV)}

Para el cálculo de las fuerzas que intervienen en la sección, se ha considerado el empuje de las bóvedas del presbiterio y
Tabla 3. Valores de cálculo para la fase 2 (siglo XIII) (Benito, 2011).

\begin{tabular}{|c|c|c|c|}
\hline & Elemento & $\begin{array}{c}\text { Componente } \\
\text { vertical } \mathrm{KN}\end{array}$ & $\begin{array}{c}\text { Componente } \\
\text { horizontal KN }\end{array}$ \\
\hline $\mathrm{F} 1$ & Pilar $(\mathrm{h}=2.42)$ & 151.44 & $\mathrm{O}$ \\
\hline F2 & Bóveda presbiterio & 286.48 & 63.69 \\
\hline $\mathrm{F}_{3}$ & $\begin{array}{l}\text { Tribuna nervio } \\
\text { superior }\end{array}$ & 7.62 & 80.81 \\
\hline $\mathrm{F}_{4}$ & Pilar $(h=10.74)$ & 672.1 & $\mathrm{O}$ \\
\hline $\mathrm{F}_{5}$ & $\begin{array}{l}\text { Bóveda girola } \\
\text { interior }\end{array}$ & 229.91 & 33.45 \\
\hline F6 & Pilar $(h=11.53)$ & 721.54 & $\mathrm{O}$ \\
\hline F7 & $\begin{array}{c}\text { Bóveda girola } \\
\text { interior }\end{array}$ & 229.91 & $33 \cdot 45$ \\
\hline F8 & $\begin{array}{l}\text { Bóveda girola } \\
\text { exterior }\end{array}$ & 320.15 & 58.64 \\
\hline F9 & Codal de piedra & $\mathrm{O}$ & 25.19 \\
\hline F10 & Pilar interior girola & 28.99 & $\mathrm{O}$ \\
\hline F11 & $\begin{array}{l}\text { Tribuna nervio } \\
\text { inferior }\end{array}$ & 302.1 & 30.87 \\
\hline F12 & $\begin{array}{c}\text { Bóveda girola } \\
\text { exterior }\end{array}$ & 320.15 & 58.64 \\
\hline $\mathrm{F} 13$ & Adarve & 177.87 & o \\
\hline F14 & Barbacana & 44.29 & o \\
\hline $\begin{array}{c}\text { F13+ } \\
\text { F14 }\end{array}$ & Fortificación & 222.16 & 140.81 \\
\hline F13 & $\begin{array}{l}\text { Muro exterior } \\
(\mathrm{h}=5.53)\end{array}$ & 1812.19 & $\mathrm{o}$ \\
\hline F14 & $\begin{array}{l}\text { Muro exterior } \\
\quad(\mathrm{h}=7.0)\end{array}$ & $2425 \cdot 5$ & o \\
\hline
\end{tabular}

de la girola, y el peso propio del pilar, estribo y fortificación. Para analizar el empuje de los arbotantes, se ha seguido la hipótesis de arbotante con empuje superior e inferior mínimo.

Para las fuerzas, se han utilizado los valores recogidos en la tabla 4:

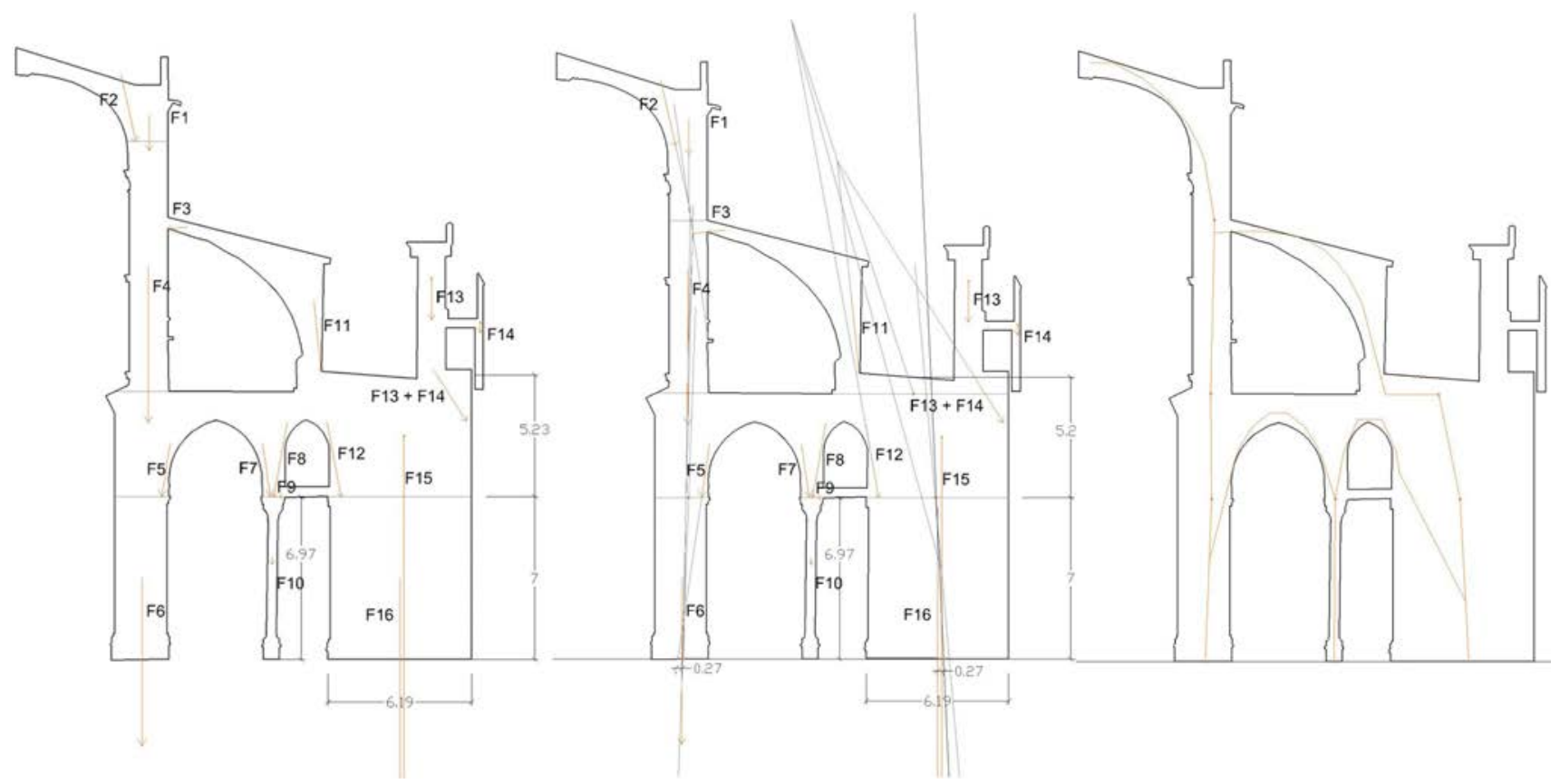

Figura 11. Distribución de fuerzas y LDE en la sección radial por el Cimorro en la opción c del siglo XIII (Benito, 2011). 
Tabla 4. Valores de cálculo para la fase 3 (siglo XIV) (Benito, 2011).

\begin{tabular}{|c|c|c|c|}
\hline & Elemento & $\begin{array}{c}\text { Componente } \\
\text { vertical } \mathrm{KN}\end{array}$ & $\begin{array}{c}\text { Componente } \\
\text { horizontal KN }\end{array}$ \\
\hline $\mathrm{F} 1$ & Pilar $(\mathrm{h}=2.42)$ & 151.44 & o \\
\hline F2 & Bóveda presbiterio & 286.48 & 63.69 \\
\hline $\mathrm{F}_{3}$ & $\begin{array}{l}\text { Arbotante superior } \\
\text { mínimo }\end{array}$ & 16.26 & 69.24 \\
\hline $\mathrm{F}_{4}$ & Pilar $(\mathrm{h}=10.74)$ & 672.1 & o \\
\hline $\mathrm{F}_{5}$ & $\begin{array}{l}\text { Arbotante inferior } \\
\text { mínimo }\end{array}$ & 15.26 & 49.86 \\
\hline F6 & $\begin{array}{l}\text { Bóveda girola } \\
\text { interior }\end{array}$ & 229.91 & 33.45 \\
\hline F7 & Pilar $(\mathrm{h}=11.53)$ & 721.54 & $\mathrm{O}$ \\
\hline F8 & $\begin{array}{l}\text { Bóveda girola } \\
\text { interior }\end{array}$ & 229.91 & 33.45 \\
\hline F9 & $\begin{array}{l}\text { Bóveda girola } \\
\text { exterior }\end{array}$ & 320.15 & 58.64 \\
\hline$F_{10}$ & Codal de piedra & O & 25.19 \\
\hline F11 & Pilar interior girola & 28.99 & o \\
\hline F12 & Estribo $(\mathrm{h}=7.35)$ & 544.85 & o \\
\hline $\mathrm{F} 13$ & $\begin{array}{c}\text { Arbotante superior } \\
\text { mínimo }\end{array}$ & 317.26 & 69.24 \\
\hline F14 & Estribo $(\mathrm{h}=4.35)$ & 336.55 & o \\
\hline $\mathrm{F} 15$ & $\begin{array}{l}\text { Arbotante inferior } \\
\text { mínimo }\end{array}$ & 107.63 & 49.86 \\
\hline $\mathrm{F} 16$ & Adarve & 177.87 & 0 \\
\hline F17 & Barbacana & 44.29 & o \\
\hline $\begin{array}{l}\text { F16+ } \\
\text { F17 }\end{array}$ & Fortificación & 222.16 & 140.81 \\
\hline $\mathrm{F} 18$ & $\begin{array}{l}\text { Muro exterior } \\
(\mathrm{h}=4.21)\end{array}$ & 1562.19 & o \\
\hline F19 & $\begin{array}{l}\text { Bóveda girola } \\
\text { exterior }\end{array}$ & 320.15 & 58.64 \\
\hline F2O & $\begin{array}{l}\text { Muro exterior } \\
(\mathrm{h}=7.0)\end{array}$ & $2425 \cdot 5$ & o \\
\hline
\end{tabular}

En la hipótesis planteada, considerando el empuje mínimo del arbotante superior y empuje mínimo del arbotante inferior, se ha calculado la intersección de la LDE con los planos de corte elegidos (fig. 12).
Análisis en la zona del pilar

El punto de intersección de la LDE con la base del pilar está separado $51 \mathrm{~cm}$ del punto medio, no incluyéndose en el tercio central de la sección. La solución es estable al quedar este incluido en la sección, pero su coeficiente de seguridad geométrico es de 1,80, bajo ya que se considera un CSG válido para estructuras góticas superior a 4.

La LDE de la sección, próxima a la cara interior del pilar, no está centrada respecto al éste, aunque sí es una solución estable al estar contenida dentro de la fábrica.

\section{Análisis en la zona del estribo}

La intersección de la LDE con la base del estribo está separada $19 \mathrm{~cm}$ del punto medio; en este caso el coeficiente de seguridad geométrico es 16 , lo que resulta un valor alto.

La LDE está centrada en el espesor del estribo, debido a la carga proporcionada por la fortificación y el momento estabilizante que introduce la barbacana en voladizo.

\section{CONCLUSIONES}

Del análisis en la opción a se concluye que es posible la construcción de la bóveda del presbiterio sin el contrarresto de la tribuna aunque en la zona del pilar interior se produce una situación comprometida de estabilidad.

Estudiando en conjunto las hipótesis a y b y las LDE en sus secciones, se concluye que la estabilidad es mucho mayor al contar con la tribuna (opción b), argumento a partir del cual podemos fechar la construcción de la tribuna en el siglo XII o principios del XIII.

Dado que en la zona de la fortificación, la situación de un basamento inferior hasta cierta altura (opción a y b) es estable sin necesitar la colocación de los adarves y la barbacana, consideramos que la construcción en el siglo XIII del adarve y la barbacana (opción c), se debió más a razones prácticas,

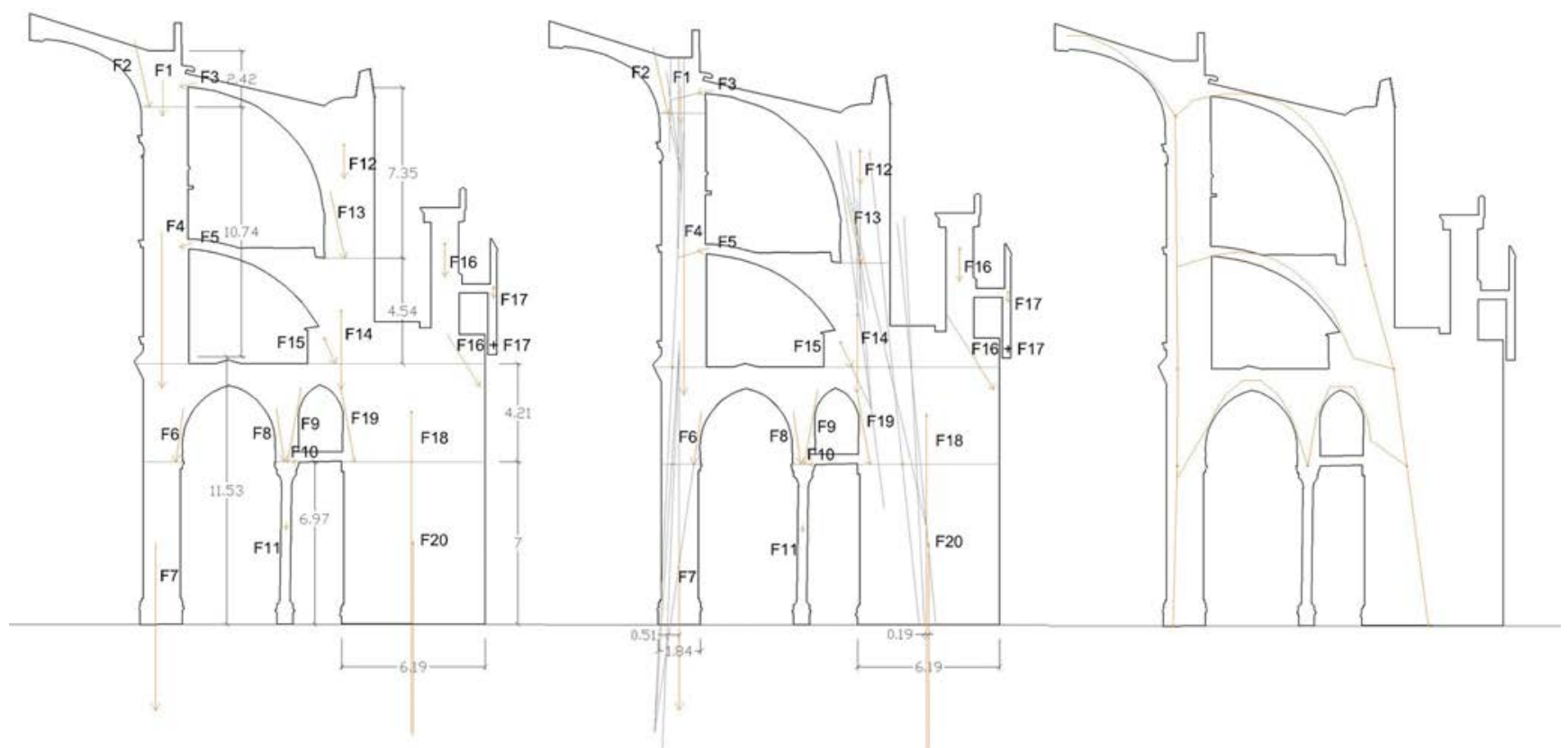

Figura 12. Distribución de fuerzas y LDE en la sección radial por el Cimorro en la opción d (siglo XIV) (Benito, 2011). 
logísticas o defensiva que a problemas estructurales de contrarresto de cargas.

La colocación de arbotantes y estribos como sistema de contrarresto (opción d), responde más a una solución compositiva de «estilo» que a una necesidad estructural. De hecho, el análisis de estabilidad en la situación anterior, con los nervios de la tribuna, tiene un mayor coeficiente de seguridad geométrico.
Entendemos que este estudio, fundamentado en el análisis estructural del edificio como fuente de información y confirmación de las hipótesis del proceso constructivo seguido en esta investigación, puede servir de referencia en futuras intervenciones, al tiempo que ayuda a completar la historia mecánica del edificio, y a comprender su comportamiento global de transmisión de cargas.

\section{REFERENCIAS}

(1) Gómez Moreno, M. (3 ed 2007). Catálogo monumental de Ávila Tomo 1. Ávila. Institución Gran Duque de Alba.

(2) Gutiérrez Robledo, J. L. (2000). Arquitectura románica y mudéjar en Ávila. Historia de Ávila 2. Edad Media (siglos VIIIXIII). Ávila. Institución Gran Duque de Alba: 517-584.

(3) Navascués Palacio, P. y Gutiérrez Robledo, J. L. (2004). La catedral de Ávila. Proceso constructivo. Las Edades del Hombre. Testigos: Santa Apostólica Iglesia Catedral, Ávila 2004. Valladolid: Fundación Las Edades del Hombre.

(4) Martín González, G. (2006). Informe sobre la zona del cimorro de la Catedral del salvador de Ávila. Inédito.

(5) Huerta Fernández, S. (2004). Arcos, Bóvedas y cúpulas. Geometría y equilibrio en el cálculo tradicional de estructuras de fábrica. Madrid. Instituto Juan de Herrera, CEHOPU.

(6) Graciani García, A. (2001). Los equipos de obra y los medios auxiliares en la Edad Media. La técnica de la Arquitectura Medieval. 1: 175-209. Sevilla. Publicaciones Universidad de Sevilla.

(7) En esta publicación se detallan todos los documentos de archivo utilizados para la realización de la hipótesis constructiva: Benito Pradillo, M A. (2016). Historia crono-constructiva de la catedral de Ávila. Ávila: Institución Gran Duque de Alba. Excma. Diputación Provincial de Ávila.

(8) Proyecto de Investigación Análisis de técnicas métricas de modelado 3d: aplicación a la reconstrucción virtual del Cimorro de la Catedral de Ávila (Universidad Católica de Ávila, 2009-2011) (Madrid De la Fuente, Carmen (IP); Benito Pradillo, Ma Ángeles; Andaluz Delgado, Soraya; Zancajo Jimeno; José Julio y Mostaza Pérez, Teresa).

(9) Heyman, J. (2001). El esqueleto de piedra. Mecánica de la arquitectura de fábrica. Madrid. Instituto Juan de Herrera.

(10) Rodríguez Almeida, E. (1975). Ensayo sobre la evolución arquitectónica de la Catedral de Ávila. Ávila. Caja Central de Ahorros y Préstamos de Ávila.

(11) Barrios García, A. (1981). Documentación Medieval de la Catedral de Ávila. Salamanca. Universidad de Salamanca.

(12) Benito Pradillo, Ma A. (2011). Evolución constructiva y análisis estructural de la Catedral de Ávila. Tesis doctoral dirigida por la Dra. Gema López Manzanares. Departamento de Estructuras de la Edificación, ETSAM, UPM. 